\title{
The characteristics of dental occlusion in patients with moderate to severe obstructive sleep apnea in Saudi Arabia
}

Nasser D. Alqahtani, BDS, MS, Mohamed I. Algowaifly, BDS, Fahad A. Almehizia, BDS, Zeyad A. Alraddadi, BDS, Fares S. Al-Sehaibany, BDS, DMSc, Naif A. Almosa, SNBO, PhD, Sahar F. Albarakati, BDS, MS, Ahmed S. Bahammam, MD, FACP.

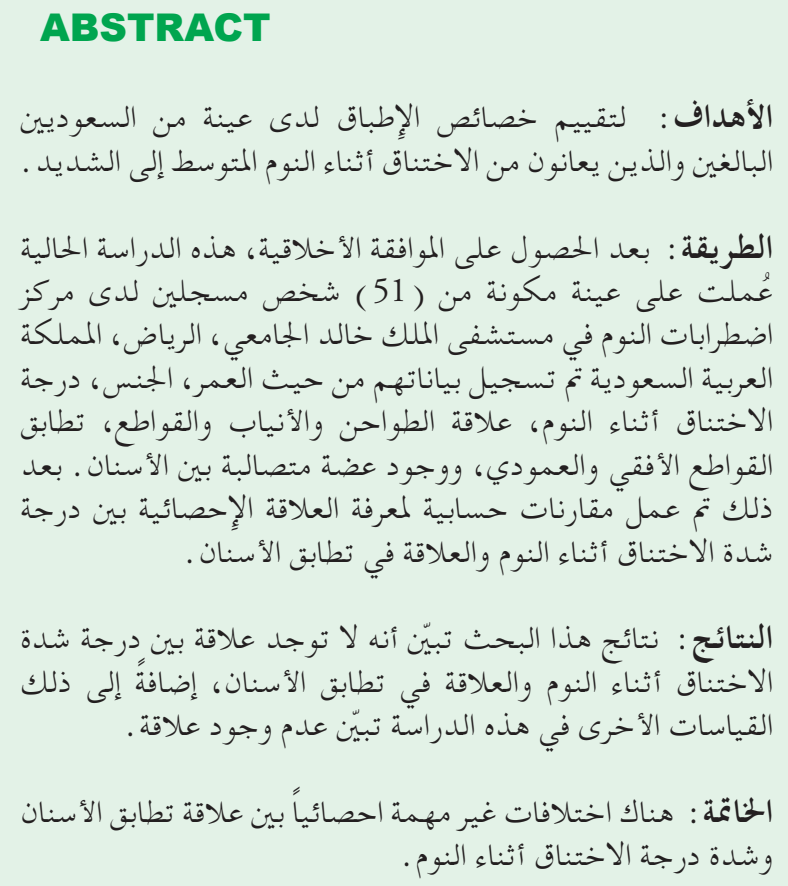

Objectives: To evaluate characteristics of dental occlusion among non-obese Saudi adult patients suffering from moderate to severe obstructive sleep apnea(OSA).

Methods: Following ethical approval, a crosssectional study was conducted at Sleep Disorders Center, King Khalid University Hospital, Riyadh, Kingdom of Saudi Arabia, between January and March 2017. Non-obese adult Saudi patients with moderate/severe OSA (apnea-hypopnea index>15) and without history of malocclusion or edentulism were included with an estimated sample size of 50 . Demographic details and severity of OSA as diagnosed by polysomnography were recorded. Characteristics of dental occlusion, namely molar, canine and incisor relationship, overjet, overbite, crossbite and arch form were obtained through calibrated examiners (kappa
0.81). Descriptive statistical analysis and Chi-square test, with $95 \%$ significance level $(p<0.05)$, were used to identify relationships between the severity of OSA and characteristics of dental occlusion.

Results: A total of 51 patients (31 males, 20 females; mean age $49.45 \pm 10.35$ years), were enrolled in the study. Severity of OSA was moderate in 17 patients and severe in 34 patients. Severe form of OSA was more among males $(64.7 \%)$ and in patients with ClassII division-1 incisor relationship (94.1\%). Neither the demographic characteristics, nor characteristics of dental occlusion showed statistically significant relationship with the severity of OSA.

Conclusion: The results of the present cross-sectional study indicate that the characteristics of dental occlusion are not related to the severity of OSA among non-obese adult Saudi patients.

Saudi Med J 2018; Vol. 39 (9):928-934

doi: 10.15537/smj.2018.9.22750

From the Department of Pediatric Dentistry and Orthodontics (Alqahtani, Al-Sehaibany, Almosa, Albarakati), College of Dentistry, from the College of Dentistry (Algowaifly, Almehizia, Alraddadi), and from the Sleep Disorders Center (Bahammam), College of Medicine, King Saud University, Riyadh, Kingdom of Saudi Arabia.

Received 29th May 2018. Accepted 10th July 2018.

Address correspondence and reprint request to: $D r$. Nasser $D$. Alqahtani, Department of Pediatric Dentistry and Orthodontics, College of Dentistry, King Saud University, Riyadh, Kingdom of Saudi Arabia. E-mail: nasserdm@ksu.edu.sa

ORCID ID: orcid.org/0000-0002-9660-9376

$\mathrm{O}$ bstructive sleep apnea (OSA) is a chronic disorder of multifactorial origin with age, gender and body mass index having been identified as some of the predisposing factors. ${ }^{1}$ While, globally the prevalence of OSA has been reported around $2-4 \%$ among the adult population, it varies greatly among different age groups, 
ranging from $4 \%$ in the middle aged, up to $80 \%$ in the geriatric population (aged 65 years and above). ${ }^{1,2}$ Similarly, the prevalence of OSA is reported to be as high as $78 \%$ among obese individuals. ${ }^{3}$ Based on a study in Saudi population, it has been reported that nearly $4.0 \%$ of adult males and $1.8 \%$ of adult females are affected by OSA. ${ }^{4}$ Evaluating the risk of OSA among Saudi population, not only was it found to be similar to that reported in Western societies, but also one out of 3 Saudi males and 4 out of 10 Saudi females are at high risk of developing OSA.4-6 Unfortunately, moderate to severe OSA still remains undiagnosed in $93 \%$ of women and $82 \%$ of men around the globe. ${ }^{1,3}$ Furthermore, undiagnosed OSA has been identified as an independent predisposing factor for early development of chronic cardiovascular, endocrine or neurological comorbid conditions. ${ }^{3}$

Clinical presentation of OSA includes intermittent and periodic reduction in respiratory airflow, which is caused due to occlusion of the upper airway during sleep. ${ }^{4}$ Excessive daytime sleepiness, loud snoring, observed breathing interruptions or gasping and choking leading to awakenings amounting to at least 5 obstructive respiratory episodes per hour during a sleep cycle are some of the reported primary symptoms. ${ }^{7}$ Patients suffering from OSA are also reported to have poor quality of sleep, daytime somnolence, cognitive disorders, hypertension, decreased productivity and increased incidence of vehicle accidents. ${ }^{8}$ On the other hand obstructive sleep apnea syndrome (OSAS) is characterized by repetitive episodes of obstructive sleep apneas and hypopneas, which is frequently followed by transient desaturation of blood oxygen levels (hypoxemia) associated with arousal from sleep. ${ }^{9}$ According to the American Academy of Sleep Medicine (AASM), the classification of OSA is based on the apnea-hypopnea index (AHI), which is defined by the average number of disordered breathing events per hour of sleep. ${ }^{3}$ Accordingly patients could be classified either as "Normal" (AHI $<5$ events per hour of sleep), "Mild OSA" (AHI $\geq 5$ and $<15$ events per hour of sleep), "Moderate OSA" (AHI $>15$ and $\leq 30$ events per hour of sleep) or "Severe OSA" (AHI>30 events per hour of sleep). ${ }^{10}$

Among the various investigations used to diagnose

Disclosure. Authors have no conflict of interests, and the work was funded by the Deanship of Scientific Research at King Saud University through Research Group no. RG-1439-54.
OSA, polysomnography (PSG) is considered as the most reliable method of detecting the frequency of apneic and hypopneic events during sleep. ${ }^{11}$ Under standardized conditions, PSG determines sleep time, stages of sleep, respiratory effort and airflow, cardiac rhythm, pulse oximetry for blood oxygen saturation, and abdominal, thoracic and limb movements through multichannel recordings. ${ }^{4}$ In addition, overnight pulse oximetry assessment has also been reported recently as a diagnostic aid equivalent to that of PSG, and with sensitivity as high as $98 \% .{ }^{12}$ Obstructive sleep apnea occurs due to a central, sleep-induced neuromuscular hypotonia, along with an associated decrease in pharyngeal space thereby resulting in inspirational collapse of upper airways. ${ }^{1}$ This obstruction leads to continuous and exacerbated respiratory efforts, apnea, hypoxemia, hypercapnia, arousal with autonomic activation and reestablishment of ventilation. ${ }^{1}$ Although repetitive arousal during sleep in patients with OSA is a major contributing factor for increased morbidity, the pathophysiology and chronicity of OSA often necessitate only symptomatic management. ${ }^{13}$

While the golden standard of management in OSA has been administrated of continuous positive airway pressure (CPAP) during sleep, modalities such as treatment with mandibular advancement device (MAD) and surgical correction of the upper airway through mandibular advancement have been useful to eliminate the underlying cause. ${ }^{14}$ Effective usage of oral appliances for mandibular advancement and management of upper airway obstruction have been reported as early as $1902 .{ }^{15}$ Evidence based studies have revealed a dramatic improvement in overall OSA symptoms, measured objectively using breathing parameters, after the prescribed usage of oral appliances (OAS)..$^{16,17}$ Interestingly, most of the oral appliances used to treat OSA are designed based on functional orthodontic appliances and are capable of exerting heavy orthodontic forces on the teeth used for anchorage. ${ }^{18}$ In spite of their ability to prevent recurrent symptoms of OSA, long term usage of oral appliances leads to clinically evident changes in the dental arch and occlusion. ${ }^{19}$

Evidences from the literature point towards an increased prevalence of OSA among obese patients. ${ }^{20-22}$ However, a direct relationship between different types of malocclusion and symptoms of OSA have been reported among non-obese adult patients. ${ }^{20,22}$ The characteristic clinical malocclusion observed among non-obese adults with OSA was predominantly, Class II malocclusion with a convex profile. $^{20}$ Similarly, cephalometric abnormalities observed among non-obese OSA patients were skeletal mandibular deficiency evidenced by a 
smaller Sella-Nasion-B point angle, short cranial base, increased lower anterior facial height, increased maxillomandibular plane angle, inferiorly positioned hyoid bone and enlarged soft palate. ${ }^{22,23}$ Surprisingly, there are no studies in the literature evaluating the characterisitcs of dental occlusion among non-obese adult patients with OSA, except for reported associations between anterior openbite, posterior crossbite and lip incompetence, and symptoms of OSA in growing patients. ${ }^{9}$ Therefore, the aim of the present study was to evaluate the relationship between characteristics of dental occlusion and severity of OSA among a cross-section of non-obese adult OSA patients in Saudi Arabia.

Methods. Ethical consideration. Following institutional ethical approval (registration number IR0132), a cross-sectional study was conducted at the "Sleep Disorders Center" at King Khalid University Hospital, Riyadh, Kingdom of Saudi Arabia, from January 2017 to March 2017. The research methodology adopted in the present study strictly adhered to the ethical standards set forth by the College of Dentistry Research Center (CDRC) and Deanship of Scientific Research, King Saud University and were in accordance with the "Helsinki declaration of 1964" and its later amendments, pertaining to studies involving human participants.

Sample selection. The sampling frame included all adult OSA patients registered with the "Sleep Disorders Center", with the exclusion criteria of obesity $(\mathrm{BMI}<30)$, previous history of dental treatment for malocclusion and edentulism (absence of a functional dentition). The sample size was estimated based on overall prevalence of OSA in Saudi population and that of malocclusion. . $-6,24^{-1}$ Calculating the sample size for the given sampling frame (Epi Info StatCalc 7, CDC, Atlanta, USA) using 95\% confidence level $(\alpha=0.05), 5 \%$ confidence interval and a statistical power of 0.85 , a study sample of 40 participants was required to achieve a statistically valid result. ${ }^{25}$ The final sample size of 50 participants $(n=50)$ was arrived after a $25 \%$ overestimation.

Non-obese adult patients with moderate or severe OSA, diagnosed based on AASM criteria, within an age range of 30-60 years were included in the study. A convenience random sampling technique was used to enroll participants, irrespective of their gender, due to the fact that the sampling frame comprised only one center in Saudi Arabia and in order to ensure recruitment of adequate number of participants to achieve outcomes which are statistically verifiable. ${ }^{25}$ Results of a standardized polysomnographic study were utilized to confirm the diagnosis of the study participants. In addition, OSA severity classification was also based on measured oxygen saturation, chest and abdominal wall movements, air flow and sleep position. The American Academy of Sleep Medicine scoring criteria was employed for manual evaluation and categorization and patients with AHI of $<15$ were excluded from the study. ${ }^{26}$

A validated data extraction tool was designed to record demographic and clinical parameters for each patient. This included the patient's age, gender, presence and severity of OSA and dental/orthodontic clinical parameters pertaining to the occlusion. Following informed consent, dental examination was carried out by 2 independently calibrated examiners, with an inter-rater agreement score (Kappa) of 0.81. The molar relationship, canine relationship, incisor relationship, horizontal incisal overlap (overjet in millimeter), vertical incisal overlap (overbite \%), cross bite (lingual or buccal) and the arch form (square, oval or narrow) were recorded.

Angle's classification (1890's), ${ }^{27}$ which divides the molar occlusion into 3 types based on the relationship between the mesiobuccal cusp of the upper permanent first molar and the buccal groove of the lower permanent first molar, was used to determine the type of molar occlusion of the participants. Similarly, the canine occlusion was determined based on the relationship between the upper permanent canine and the interdental embrasure between the lower permanent canine and lower first premolar. ${ }^{27}$ In order to determine the incisor occlusion between the upper and lower permanent anterior teeth, the "British Standards Institute's incisor classification of malocclusion" was used. ${ }^{28}$

Statistical analysis. The collected data was entered in a spreadsheet software (Microsoft EXCEL 2010) and exported to a statistical software package (IBM SPSS Statistics Version 21, IBM Corp., Armonk, NY, USA). The data was subjected to descriptive statistical analysis. Measures of central tendency and dispersion were calculated for the numerical variables, namely age of the patients, overbite $(\%)$ and overjet $(\mathrm{mm})$. Frequencies and percentages were calculated for the nominal variables. Non-parametric Chi-square test was utilized for further analysis of data. A 95\% significance level $(p<0.05)$ was assumed for all statistical analysis.

Results. A total of 51 patients $(31$ males and 20 females) with a mean age of $49.45 \pm 10.35$ years (range 36-60 years), who fulfilled the inclusion criteria, were enrolled in the present study. While all the patients were diagnosed previously with OSA, 17 patients had moderate OSA and 34 patients had 
severe OSA. Although descriptive analysis (Table 1) revealed predominance of severe form of OSA among males $(64.7 \%)$, there was no statistically significant relationship between severity of OSA and gender of the participants $(p=0.417)$. Similarly, the age of the patients was also not significantly associated with the severity of OSA $(p=0.236)$.

Based on clinical evaluation, majority of the examined OSA patients had Angle's Class II molar (43.1\%) and canine $(49.0 \%)$ occlusion (Figure 1). Nevertheless, there was no statistically significant relationship between the molar $(p=0.701)$ and canine $(p=0.328)$ occlusion to that of the severity of OSA. Similarly, there was no statistically significant relationship between the observed "British Standards Institute's incisor classification of malocclusion" and the severity of OSA. However, it was an interesting observation that while only $45.1 \%$ of the OSA patients had Class I incisor relationship $(p=0.280)$, nearly $94.1 \%$ of the OSA patients had Class II division 1 incisor relationship ( $p=0.058$ ) (Figure 1).

There was no statistically significant relationship between the severity of OSA of the studied patients and the observed degrees of overjet $(p=0.427)$, overbite $(p=0.861)$ and buccal or lingual crossbite $(p=0.364)$. Although $58.8 \%$ of the observed OSA patients
Table 1 - Comparison of demographic characteristics between patients with moderate and severe obstructive sleep apnea.

\begin{tabular}{|c|c|c|}
\hline \multirow[t]{2}{*}{ Demographic characteristics } & \multicolumn{2}{|c|}{ Severity of obstructive sleep apnea } \\
\hline & Moderate $\mathrm{n}=17$ & Severe $n=34$ \\
\hline Gender & \multicolumn{2}{|c|}{ n $(\%)$} \\
\hline Male & $9(52.9)$ & $22(64.7)$ \\
\hline Female & $8(47.1)$ & $12(35.3)$ \\
\hline Age Mean \pm SD & $47.65 \pm 11.264$ & $51.24 \pm 9.429$ \\
\hline
\end{tabular}

manifested oval arch forms and $31.4 \%$ of them had narrow arches, there was no statistically significant relation between the type of arch form and severity of OSA ( $p=0.769)$ (Figure 2). Overall, the findings of the present cross-sectional study did not reveal any statistically significant relationship between the characteristics of dental occlusion and severity of OSA in non-obese adult patients.

Discussion. The present cross-sectional study evaluated the characteristics of dental occlusion among non-obese adult Saudi patients with moderate to severe OSA, registered with the Sleep Disorders Center at King Khalid University Hospital. Not only is altered craniofacial anatomy considered an important etiological

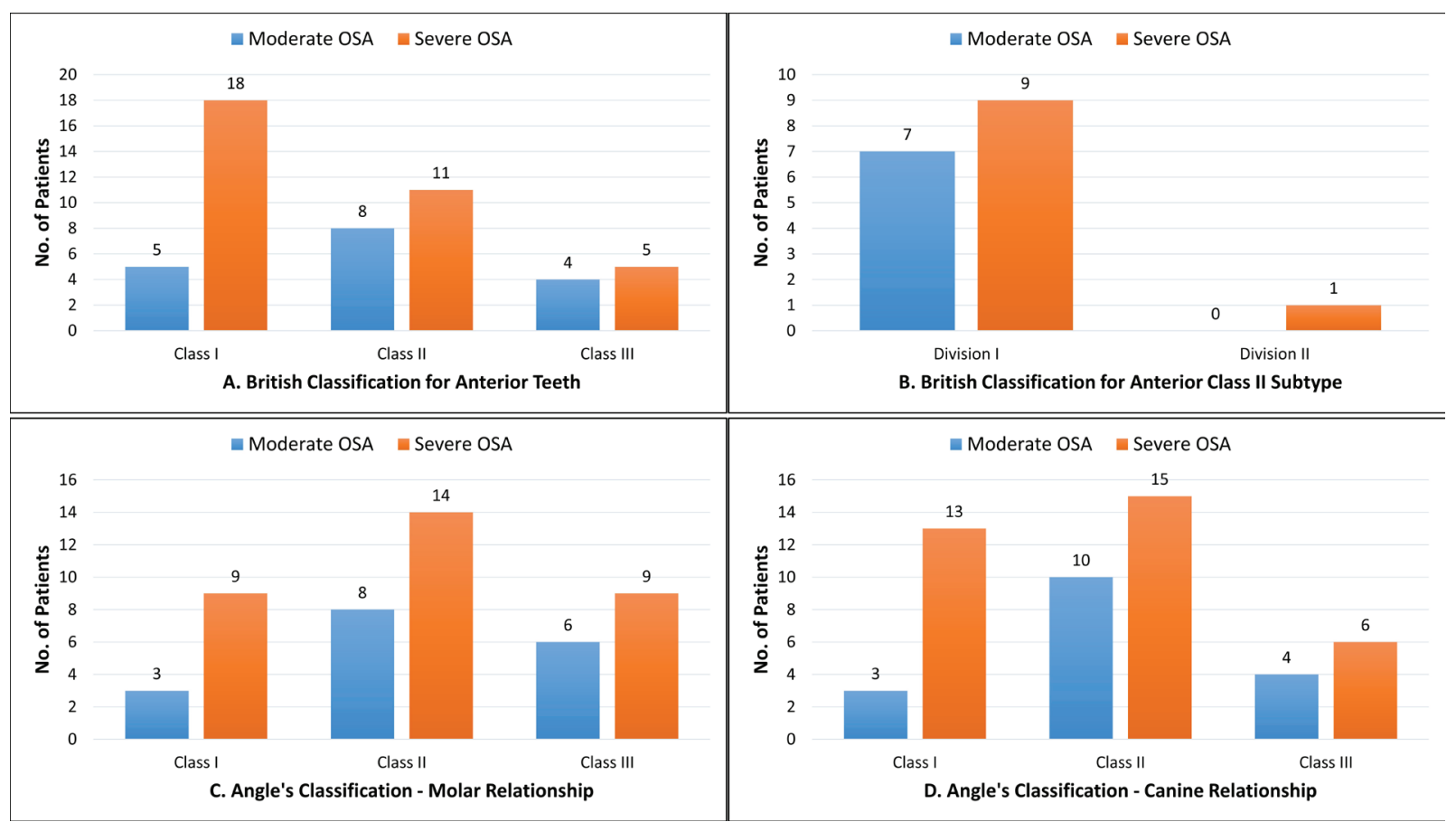

Figure 1 - Bar graphs showing frequency distribution of patient with moderate and severe obstructive sleep apnea in relation to A) British classification of anterior teeth; B) British classification of anterior class II subtype; C) Angle's classification - molar relationship and D) Angle's classification canine relationship. 


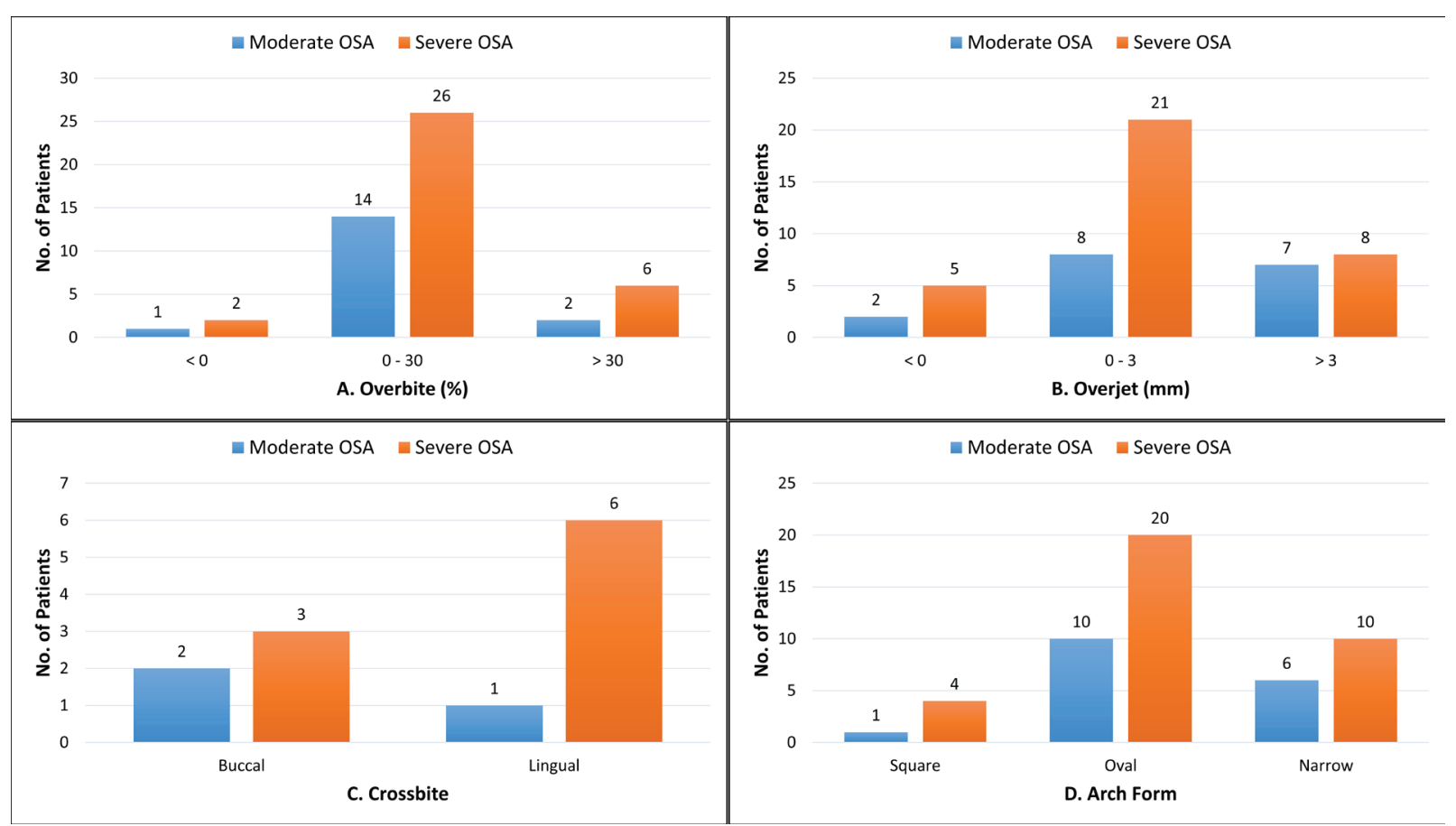

Figure 2 - Bar graphs showing frequency distribution of patient with moderate and severe obstructive sleep apnea in relation to: A) Overbite; B) Overjet; C) Crossbite and D) Arch form.

factor in patients with OSA, but also the presence of skeletal malocclusion is a major predisposing factor for the development of OSA symptoms, especially among non-obese patients. ${ }^{20,22,29}$ Interestingly, there are no studies in the literature evaluating dental malocclusion and its associations with the risk of developing OSA, except in growing patients. ${ }^{9}$ Considering the fact that the prevalence of OSA and the risk of developing OSA among Saudi adult population is almost similar to that of other population groups worldwide, the present study endeavored to evaluate any potential association between the characteristics of dental occlusion and the severity of OSA. ${ }^{4-6}$

The observed data in this study did not demonstrate any considerable association between the different characteristics of molar, canine and incisor occlusion, with that of the severity of OSA. Although these findings are contrary to what has been reported earlier regarding the relationship between Class III malocclusion and the risk of developing symptoms of OSA, they could have been attributed to skeletal Class III tendencies. ${ }^{23}$ While dental malocclusion, presenting clinically as increased overbite and posterior crossbite, has been implicated in OSA symptoms among growing patients, no such associations were observed in the present study among non-obese adult patients.
In a study reported by Lee et $\mathrm{al},{ }^{8}$ it was found that the morbidity of OSA patients was directly related to its severity. Even though evidence based practices and AASM levels of recommendations advocate that occlusal changes are not always associated with the severity of OSA, extensive diagnostic protocols must be followed including occlusal discrepancies before initiating treatment with oral appliances in order to identify the ideal patient population with risk of complications and associated dental comorbidities. ${ }^{18}$ In previous studies, it has been reported that Class II malocclusion is closely associated with the severity of OSA, which could be attributed to a backward positioning of the tongue in such patients. ${ }^{20,30}$ This finding was in disagreement with the outcome of the present study, as the severity of OSA did not have any association with class II molar or canine occlusion. This difference could be due to issues related to ethnicity, as some racial groups present predominantly with Class I malocclusion in OSA conditions. ${ }^{31}$ Nevertheless, Class II division 1 incisor relationship was associated with a greater, but nonsignificant, number of patients with severe OSA in the present study.

During the examination for recording overbite and overjet, it was found that the OSA patients did not have deep overbite or severe overjet as was reported by other 
studies. ${ }^{24}$ Maximum number of patients with moderate to severe OSA presented with overbite ranging from $0-30 \%$ and over-jet of $0-3 \mathrm{~mm} .{ }^{24}$ Buccal or lateral cross-bite due to maxillary skeletal constriction were found to be present in approximately $30-50 \%$ of OSA patients irrespective of the severity measured. ${ }^{24}$ However, the present study demonstrated that the OSA patients were commonly associated with anterior cross-bite. An appreciable difference in distribution of cross-bite was observed among the patients with differing OSA severity but was not statistically significant.

The shape of the dental arch which is obtained by configuration of the supporting bone after the eruption of the teeth, the circum-oral musculature and intra-oral forces are of esthetic and functional importance. ${ }^{13}$ In the present study, the oval arch shape was dominant in both the OSA severity patients followed by square and narrow arch forms. The oval arch form accounted for almost $60 \%$ of the total arch forms which was similar to the findings of Acar et al. ${ }^{32}$ Overall, the results of the present study are in concurrence with the fact that, some patients may suffer from OSA although they have normal occlusion, as was found in the majority of the studied participants who had normal sagittal and vertical occlusal relationships, thereby attributing OSA to non-dental factors. ${ }^{8}$

One of the major limitations of the present study was the use of a simple method of dental examination, without the analysis of either cephalometric radiographs or study models and hence could be utilized as a standard of initial assessment at best. Moreover, the aim of the present study was to assess the relationship between dental occlusion and severity of OSA among non-obese patients, as a result of which the BMI of the patients was not considered as a variable for evaluation. Nevertheless, the outcomes of the present study could help in further studies and enlighten the clinicians of the possible relationships between dental occlusion and the severity of OSA.

In conclusion, based on the results of the present study, the different characteristics of dental occlusion have no relationship to the severity of OSA among non-obese adult patients in a subset of Saudi populations. However, the outcomes of the study need to be further validated by evaluation in a larger sample size derived out of differing ethnic groups, before it could be extrapolated on a global scale.

Acknowledgment. The authors extend their appreciation to the Deanship of Scientific Research at King Saud University for funding this work through Research Group no. RG-1439-54

\section{References}

1. Jordan AS, McSharry DG, Malhotra A. Adult obstructive sleep apnoea. Lancet 2014; 383: 736-747.

2. Kleisiaris CF, Kritsotakis EI, Daniil Z, Tzanakis N, Papaioannou A, Gourgoulianis KI. The prevalence of obstructive sleep apneahypopnea syndrome-related symptoms and their relation to airflow limitation in an elderly population receiving home care. Int J Chron Obstruct Pulmon Dis 2014; 9: 1111-1117.

3. Park JG, Ramar K, Olson EJ. Updates on definition, consequences, and management of obstructive sleep apnea. Mayo Clin Proc 2011; 86: 549-554; quiz 554-555.

4. Wali SO, Abalkhail B, Krayem A. Prevalence and risk factors of obstructive sleep apnea syndrome in a Saudi Arabian population. Annals of thoracic medicine 2017; 12: 88-94.

5. Bahammam AS, Al-Rajeh MS, Al-Ibrahim FS, Arafah MA, Sharif MM. Prevalence of symptoms and risk of sleep apnea in middle-aged Saudi women in primary care. Saudi Med J 2009; 30: 1572-1576.

6. BaHammam AS, Alrajeh MS, Al-Jahdali HH, BinSaeed AA. Prevalence of symptoms and risk of sleep apnea in middle-aged Saudi males in primary care. Saudi Med J 2008; 29: 423-426.

7. Ferguson KA, Cartwright R, Rogers R, Schmidt-Nowara W. Oral appliances for snoring and obstructive sleep apnea: a review. Sleep 2006; 29: 244-262.

8. Lee JE, Lee CH, Lee SJ, Ryu Y, Lee WH, Yoon IY, et al. Mortality of patients with obstructive sleep apnea in Korea. $J$ Clin Sleep Med 2013; 9: 997-1002.

9. Caprioglio A, Meneghel M, Fastuca R, Zecca PA, Nucera R, Nosetti L. Rapid maxillary expansion in growing patients: correspondence between 3-dimensional airway changes and polysomnography. Int J Pediatr Otorhinolaryngol 2014; 78: 23-27.

10. Epstein LJ, Kristo D, Strollo PJ Jr, Friedman N, Malhotra A, Patil SP, et al. Clinical guideline for the evaluation, management and long-term care of obstructive sleep apnea in adults. J Clin Sleep Med 2009; 5: 263-276.

11. AlRumaih HS, Baba NZ, AlShehri A, AlHelal A, Al-Humaidan A. Obstructive Sleep Apnea Management: An Overview of the Literature. J Prosthodont 2018; 27: 260-265.

12. Lin CL, Yeh C, Yen CW, Hsu WH, Hang LW. Comparison of the indices of oxyhemoglobin saturation by pulse oximetry in obstructive sleep apnea hypopnea syndrome. Chest 2009; 135 : 86-93.

13. Ngiam J, Balasubramaniam R, Darendeliler MA, Cheng AT, Waters K, Sullivan CE. Clinical guidelines for oral appliance therapy in the treatment of snoring and obstructive sleep apnoea. Aust Dent J 2013; 58: 408-419.

14. Knappe SW, Sonnesen L. Mandibular positioning techniques to improve sleep quality in patients with obstructive sleep apnea: current perspectives. Nat Sci Sleep 2018; 10: 65-72.

15. Greenstone M, Hack M. Obstructive sleep apnoea. BMJ 2014; 348: g3745.

16. Sutherland K, Chan AS, Cistulli PA. Three-dimensional assessment of anatomical balance and oral appliance treatment outcome in obstructive sleep apnoea. Sleep Breath 2016; 20 : 903-910.

17. Gakwaya S, Melo-Silva CA, Borel JC, Rousseau E, Masse JF, Sériès F. Impact of stepwise mandibular advancement on upper airway mechanics in obstructive sleep apnea using phrenic nerve magnetic stimulation. Respir Physiol Neurobiol 2014; 190: 131-136. 
18. Ramar K, Dort LC, Katz SG, Lettieri CJ, Harrod CG, Thomas $\mathrm{SM}$, et al. Clinical Practice Guideline for the Treatment of Obstructive Sleep Apnea and Snoring with Oral Appliance Therapy: An Update for 2015. J Clin Sleep Med 2015; 11 : 773-827.

19. Sharples LD, Clutterbuck-James AL, Glover MJ, Bennett MS, Chadwick R, Pittman MA, et al. Meta-analysis of randomised controlled trials of oral mandibular advancement devices and continuous positive airway pressure for obstructive sleep apnoea-hypopnoea. Sleep Med Rev 2016; 27: 108-124.

20. Banabilh SM, Samsudin AR, Suzina AH, Dinsuhaimi S. Facial profile shape, malocclusion and palatal morphology in Malay obstructive sleep apnea patients. Angle Orthod 2010; 80: $37-42$.

21. Lopez PP, Stefan B, Schulman CI, Byers PM. Prevalence of sleep apnea in morbidly obese patients who presented for weight loss surgery evaluation: more evidence for routine screening for obstructive sleep apnea before weight loss surgery. Am Surg 2008; 74: 834-838.

22. Miyao E, Noda A, Miyao M, Yasuma F, Inafuku S. The role of malocclusion in non-obese patients with obstructive sleep apnea syndrome. Intern Med 2008; 47: 1573-1578.

23. Lavezzi AM, Casale V, Oneda R, Gioventù S, Matturri L, Farronato G. Obstructive sleep apnea syndrome (OSAS) in children with Class III malocclusion: involvement of the PHOX2B gene. Sleep Breath 2013; 17: 1275-1280.

24. Gudipaneni RK, Aldahmeshi RF, Patil SR, Alam MK. The prevalence of malocclusion and the need for orthodontic treatment among adolescents in the northern border region of Saudi Arabia: an epidemiological study. BMC Oral Health 2018; 18: 16.
25. Ramalingam S, Habib SR, Sundar C, Dawas AB, Al-Rashed M, Al-Bader R. Perceptions of dental interns in Saudi Arabia toward implant placement in medically compromised patients. J Educ Health Promot 2017; 6: 104.

26. Berry RB, Brooks R, Gamaldo CE, Harding SM, Lloyd RM, Marcus CL, et al. The AASM Manual for the Scoring of Sleep and Associated Events: Rules, Terminology and Technical Specifications. Darien (IL): American Academy of Sleep Medicine; 2012.

27. Angle EH. Treatment of Malocclusion of the Teeth and Fractures of the Maxillae: Angle's System: Philadelphia (PA): BiblioLife; 2015.

28. Arvind TV, Baskaranarayanan B, Chidambaram S, Premkumar S. Association between the British Standards Institute's incisor classification of malocclusion and Angle's Classification of malocclusion-an analytical study. RGUHS Journal of Medical Sciences 2015; 5: 62-66.

29. Marcus CL, Brooks LJ, Draper KA, Gozal D, Halbower AC, Jones J, et al. Diagnosis and management of childhood obstructive sleep apnea syndrome. Pediatrics 2012; 130: e714-755.

30. Banabilh SM. Orthodontic view in the diagnoses of obstructive sleep apnea. J Orthod Sci 2017; 6: 81-85.

31. Patel N, Nagle M. Having an angle class II malocclusion is associated with a higher incidence of obstructive sleep apnea: An evidence-based synopsis. San Antonio (TX): UT HSCSA CATs Library CAT; 2013. Available from: https://cats.uthscsa. edu/found_cats_view.php?id=2364

32. Acar M, Türkcan İ, Özdaș T, Bal C, Cingi C. Obstructive sleep apnoea syndrome does not negatively affect oral and dental health. J Laryngol Otol 2015; 129: 68-72. 